\title{
EXPERIENCIA INTERNA Y SUBJETIVIDAD EN LA REFUTACIÓN KANTIANA DEL IDEALISMO PROBLEMÁTICO
}

ClAUdDIA JÁUREgUI

UNIVERSIDAD DE BUENOS AIRES

1. Introducción. Algunas consideraciones sobre la relación entre la experiencia interna y la externa en el cuarto paralogismo de la razón pura

La doctrina kantiana del sentido interno ha sido considerada frecuentemente como uno de los puntos tal vez más oscuros del idealismo trascendental. El interés de Kant parece concentrarse, tanto en la Crítica de la razón pura $(\mathrm{KrV})$ como en gran parte de sus otras obras, en determinar cuáles son las condiciones que hacen posible la experiencia, pero entendiendo particularmente esta última como experiencia de los objetos en el espacio. El problema de las condiciones que se requieren para el conocimiento introspectivo de nosotros mismos aparece, en líneas generales, relegado a un segundo plano y no queda claro hasta qué punto es posible una autobjetivación del yo.

En ocasiones Kant sugiere que existe un paralelismo entre el sentido interno y el sentido externo. El entendimiento humano es finito y no puede, por tanto, conocer a menos que el objeto le sea dado por medio de la sensibilidad. La experiencia supone cierta receptividad del sujeto por la cual es posible que los objetos se nos hagan presentes. Pero no sólo somos receptivos respecto de los objetos físicos, sino que también somos receptivos en relación con nosotros mismos. Por medio del sentido interno nos autocaptamos no tal como somos sino tal como aparecemos. El yo es pues para sí un fenómeno del mismo modo en que lo son los objetos en el espacio. Si esto es así, podríamos decir, en principio, que existe una simetría entre la experiencia interna y la externa. En ambos casos, los objetos se nos hacen presentes mediante la sensibilidad. La única diferencia residiría pues en que, en la primera, el objeto del sentido interno, es decir, el yo empírico, aparece bajo la forma del tiempo, mientras que en la segunda, el objeto del sentido externo, es decir, el objeto físico, aparece espacialmente, o si se quiere tempo-espacialmente.

Este paralelismo entre el sentido interno y el sentido externo le permite a Kant refutar, en el capítulo de los "Paralogismos de la razón pura" de 
la primera edición de la $K r V$ (cfr. A 367-380), el idealismo problemático cartesiano según el cual hay una asimetria entre la conciencia de nuestra propia existencia y la de la existencia de los objetos exteriores, en el sentido de que la primera goza de absoluta certeza y se nos da en forma inmediata en la percepción interna, mientras que la segunda, por el contrario, se nos presenta siempre dudosa. En efecto, el idealista cartesiano parte de la premisa de que sólo podemos percibir inmediatamente aquello que está en nosotros mismos y, en este sentido, sólo nuestra propia existencia puede ser objeto de percepción. La existencia de los objetos que están fuera de nosotros nunca es percibida directamente, sino que es inferida meramente como la causa de aquella modificación del sentido interno que llamamos percepción.

Kant refuta, en el capítulo de los paralogismos, esta tesis cartesiana reinterpretando, a la luz del idealismo trascendental, la expresión "objeto fuera de nosotros" u "objeto exterior". El idealista problemático se apoya, a su entender, en presupuestos gnoseológicos falsos desde el momento en que considera que los objetos exteriores son cosas en sí que existen independientemente de nuestras representaciones. Una vez que la cuestión se plantea de esta manera, es congruente concluir que la existencia de los objetos exteriores es incierta, ya que no hay modo de acceder a los mismos más que por medio de las representaciones que tenemos de ellos, y subsiste, por tanto, siempre la duda acerca de la existencia de una realidad que trascienda nuestros estados internos de conciencia. Si adoptamos, por el contrario, el idealismo trascendental que Kant propone, debemos considerar que los objetos exteriores no son otra cosa más que los objetos espaciales, es decir, que no son independientes de nuestra sensibilidad, sino que constituyen más bien un tipo de representación. Tenemos así representaciones internas por las cuales tomamos conciencia de nosotros mismos y representaciones externas por las cuales somos conscientes de los fenómenos en el espacio. En ambas situaciones, la percepción nos presenta un objeto en forma inmediata cuya existencia goza, en los dos casos, del mismo grado de certeza. Kant traslada, de este modo, el dualismo entre el yo y la materia, entendidos como cosas en sí, al plano fenoménico; y los interpreta como dos tipos de representaciones cuya existencia, en tanto representaciones, resulta indubitable. Desaparece pues desde la óptica del idealismo trascendental la radical asimetría que el cartesianismo establecía entre la experiencia interna y la externa, y el supuesto acceso privilegiado que tendríamos al conocimiento de nuestra propia existencia.

Creemos, sin embargo, que este argumento que Kant propone en el capítulo de los paralogismos no logra en sentido estricto refutar el idealismo problemático. La distinción entre dos tipos de representaciones -las espaciales y las temporales- cuyos objetos respectivos se presentan con el mismo grado de inmediatez y certeza no parece ser suficiente para superar 
el solipsismo. Cabría la posibilidad de que los objetos físicos fueran imaginados o soñados meramente. ${ }^{1} \mathrm{El}$ hecho de tener representaciones espaciales no permitiría, en principio, asegurar que existe algo diferente de nosotros mismos. Dichas representaciones podrían no ser otra cosa más que meros estados subjetivos de conciencia y sería posible, por ende, reducir la experiencia externa a la interna. Al equiparar ambos lados de la experiencia en cuanto al carácter fenoménico que respectivamente presentan sus objetos, se debilitan, a nuestro entender, los criterios que permiten diferenciar la autoconciencia empírica de nosotros mismos de la conciencia empírica de aquello que no somos nosotros. El argumento de los paralogismos parece reducir la distinción entre la experiencia interna y la externa a una mera diferencia entre dos ámbitos de representaciones -las temporales y las espaciales-, con lo cual podría suceder perfectamente que sólo existieran el yo y estas representaciones y que los objetos físicos, en cuanto objetos representados, no fueran otra cosa más que estados internos del sujeto mediante los cuales el yo se volvería, a la vez, empíricamente autoconsciente de sí mismo. Se daría, de este modo, una superposición entre el sentido interno y el externo, y el idealismo trascendental se transformaría en un subjetivismo extremo, el cual, más que superar el idealismo problemático cartesiano, lo reproduciría a partir de otros presupuestos gnoseológicos.

Cabe señalar que en el mismo capítulo de los paralogismos (cfr. A 374 375), Kant propone un argumento, complementario del que analizamos anteriormente, tendiente a mostrar que los objetos físicos no pueden ser sólo un producto de nuestra fantasía. Así como el espacio es la representación de la mera posibilidad de la coexistencia, la percepción es la representación de una realidad. Esta realidad, en el caso de los objetos exteriores, es representada en el sentido externo, es decir, en el espacio. Ahora bien, el espacio en sí mismo no es más que una mera representación; así pues, en él sólo puede considerarse real lo que en él se representa, e inversamente lo que está dado en él, es decir, lo representado por medio de la percepción es también lo real en él. En consecuencia, toda percepción externa constituye la prueba inmediata de algo real en el espacio.

En este nuevo argumento, la percepción aparece como la garantía de la realidad (Wirklichkeit) de los objetos exteriores. La existencia de estos últimos no sólo queda asegurada por el reconocimiento de su carácter fenoménico, sino también por el modo perceptual en que son dados en la intuición. Podríamos soñar o imaginar objetos espaciales, sin embargo, la imaginación y el sueño - dice Kant- presuponen siempre la percepción (cfr. A 374 y A 377). Para evitar este tipo de ilusiones, debemos considerar que es real todo aquello que está dado en la percepción o está conectado con la misma según leyes empíricas (cfr. A 376).

1 Sobre esta cuestion cfr. M. Hymers, "The Role of Kant's Refutation of Idealism", The Southern Journal of Philosophy, vol. XXIX, no. 1, 1991. 
Creemos que este segundo argumento basado en la noción de "percepción" y su conexión con la noción de "realidad" no es tan convincente como el primero que Kant presenta en el texto del cuarto paralogismo. Por una parte, no se comprende cómo se justifica la afirmación de que la imaginación y el sueño presuponen la percepción. Y en segundo lugar, la realidad de los objetos físicos parece fundarse sólo en el hecho de que son representados perceptualmente. Aun suponiendo que no fueran imaginados o soñados, tal percepción podría no ser otra cosa más que un mero estado subjetivo de conciencia; con lo cual se produciría nuevamente una confusión entre el sentido interno y el externo y quedaríamos, una vez más, atrapados en una postura subjetivista. Para refutar el idealismo problemático es preciso, a nuestro entender, establecer un criterio de distinción entre la experiencia interna y la externa que no radique meramente en la forma sensible en que aparecen sus respectivos fenómenos, sino que permita trazar además claramente la diferencia entre dos órdenes: el de mis representaciones internas, es decir, mis representaciones entendidas como estados subjetivos de conciencia a los cuales sólo yo tengo acceso y el de las representaciones de un mundo físico, intersubjetivo y, por así decirlo, público. Y esta distinción deberá establecerse además de modo tal que el segundo orden sea irreductible al primero. Dicho con otras palabras, el ámbito de los objetos físicos de alguna manera debería "trascender" o. "exceder" el ámbito de mis estados psíquicos, sin que esto signifique, de ningún modo, negar el carácter fenoménico de los objetos espaciales o pretender alcanzar el conocimiento de un mundo de cosas en sí.

Creemos que si nos mantenemos dentro del marco conceptual del idealismo trascendental, esta distinción entre ambos órdenes sólo puede establecerse sobre la base de alguna diferencia en el modo de aplicación de las categorías en cada uno de ellos.

A continuación intentaremos demostrar que, si bien Kant no desarrolló expresamente esta estrategia argumentativa contra el idealismo problemático, la misma está, sin embargo, implícita en el pasaje agregado en la segunda edición de la $\mathrm{KrV}$, titulado la "Refutación del idealismo". Allí Kant propone un nuevo argumento contra el cartesianismo en el cual, una vez más, la diferencia entre la experiencia interna y la externa parece residir meramente en los caracteres formales sensibles que una y la otra presentan respectivamente. En este caso, ya no se pone tanto el acento en el hecho de que los fenómenos de la primera aparecen en el tiempo, mientras que los de la segunda se muestran en el espacio, sino que se establecerá precisamente una distinción entre los rasgos temporales propios de cada una. Sin embargo, los caracteres temporales atribuidos a la experiencia interna determinan - como trataremos de probar más adelante- que algunas de las categorías hallen difícil aplicación en este ámbito de representaciones, con lo cual se plantea el problema de que el yo no podría ser considerado 
un objeto genuino, ya que la multiplicidad intuitiva a partir de la cual sería menester constituirlo no se sujetaría estrictamente a aquellas condiciones que hacen posible la objetividad. La experiencia interna se revelaría, por tanto, como una pseudoexperiencia y sólo podríamos calificar, en rigor, como algo objetivo el conocimiento de los objetos físicos.

Los caracteres temporales atribuidos respectivamente a la experiencia externa y a la interna en la "Refutación del idealismo" determinarían pues la posibilidad de establecer un criterio de distinción entre ambas que no residiría meramente en las diferentes formas sensibles que sus fenómenos muestran, sino que permitiría contrastar además dos órdenes de representaciones con distinto grado de objetividad y, como veremos más adelante, permitiría, por otra parte, contrastarlos de modo tal que los fenómenos físicos no puedan quedar reducidos, en ningún caso, a meros fenómenos psíquicos.

\section{El argumento de la "Refutación del idealismo"}

El punto de partida de esta nueva argumentación dirigida contra el idealismo problemático consiste en la afirmación de aquello que Descartes acepta como algo absolutamente cierto, es decir, que tenemos experiencia interna. No hay duda de que somos empíricamente autoconscientes de nuestra propia existencia y esto significa, de acuerdo con el idealismo trascendental, que tenemos conciencia de nuestra propia existencia como algo determinado en el tiempo ( $c f r$. B 275). Cabe recordar aquí que para Kant hay dos formas distintas de autoconciencia - la apercepción trascendental y la apercepción empírica- y, en consecuencia, dos maneras también de acceder a nuestra propia existencia que el cartesianismo no diferencia entre sí. Sabemos, en efecto, por la "Deducción trascendental" (B) que la posibilidad de la unidad sintética de la multiplicidad se funda en que el "yo pienso" debe poder acompañar todas las representaciones, de modo tal que pueda llamarlas a todas ellas mías (B 131-132). Esta identidad del yo es, sin embargo, una representación vacía de contenido en la cual se me da, al mismo tiempo, la autoconciencia de mi propia existencia pero de un modo indeterminado. Soy consciente de que soy, pero no puedo saber cómo soy. Esta representación es un mero pensamiento y, como no hay en nosotros intuición intelectual, el "yo pienso/yo soy" no proporciona ningún contenido mediante el cual pueda determinar mi propia existencia y decir que existo de tal o cual manera. Para que tal determinación tenga lugar, se requiere una intuición que en nosotros no puede ser más que sensible. El sentido interno es pues el encargado de procurar el material intuitivo que da contenido al "yo soy". Accedemos a nuestra propia existencia por medio de la sensibilidad interna y, siendo esta última temporal, la conciencia determinada de nuestra existencia no podrá darse más que en el tiempo. Existe pues, junto con la apercepción trascendental, una autoconciencia empírica de nosotros 
mismos y es a esta última a la que hace referencia la primera premisa del argumento de la "Refutación del idealismo".

La segunda premisa afirma que toda determinación en el tiempo presupone algo permanente en la percepción. En efecto, tenemos conciencia de nosotros mismos por medio de una serie de estados subjetivos siempre cambiantes. Pero para que el cambio se constituya como tal, es necesario un sustrato permanente con respecto al cual la multiplicidad interna se determine sucesiva. ${ }^{2}$ Esto permanente, agrega Kant, no puede ser una intuición en $\mathrm{mi}(c f r . \mathrm{B} \mathrm{XL}){ }^{3}$ Todo lo que encuentro en el sentido interno son representaciones siempre cambiantes que requieren algo permanente, diferente de ellas mismas, que haga posible la determinación de mi existencia en el tiempo. En lo que llamamos "alma" todo está en continuo flujo y no hay en ella absolutamente nada que permanezca (cfr. A 381). ${ }^{4}$

Ahora bien, si esto es así, aquello permanente cuya percepción hace posible la determinación de mi existencia en el tiempo debe ser un objeto en el espacio. La autoconciencia empírica de mi propia existencia está ligada necesariamente a la conciencia de la condición que hace posible su determinación en el tiempo y esta condición es precisamente la existencia de los objetos exteriores. La autoconciencia empírica de mi existencia es pues, a la vez, una conciencia inmediata de la existencia de las cosas fuera de mí (cfr. B 276). En este punto, Kant introduce una importante modificación respecto del argumento contra el idealismo problemático que aparecía en los "Paralogismos de la razón pura". Mientras que allí se rompía la asimetría entre la experiencia externa y la interna mostrando que la primera era tan inmediata como la segunda, en el argumento de la "Refutación del idealismo" se reestablece la asimetría pero en un sentido exactamente inverso a aquel que le atribuía el idealismo cartesiano. La experiencia interna y el acceso a nuestra propia existencia, lejos de ser lo más inmediato, aparecen ahora, en este nuevo argumento, mediados por la experiencia externa. Es esta última la que goza de aquella inmediatez y certeza que el cartesianismo

2 La necesidad de un sustrato permanente como condición de posibilidad de las determinaciones temporales constituye la tesis central de la primera analogía de la experiencia (cfr. A 182 = B 224 y ss.).

3 El texto original dice: "Esto permanente no puede, sin embargo, ser algo en mí, ya que es sólo a través de esto permanente que mi existencia misma puede ser determinada en el tiempo" (B 275). Kant pide en el prefacio (B) que este pasaje sea modificado para su mejor comprensión. En nuestro análisis, nos basaremos en la versión corregida del texto.

4 Este modo de entender la experiencia interna se asemeja a aquel que encontramos en el Tratado de la naturaleza humana (libro I, parte IV, sec. VI). Allí Hume afirma que: "La mente es una especie de teatro en el que distintas percepciones se presentan en forma sucesiva; pasan, vuelven a pasar, se desvanecen y mezclan en una variedad infinita de posturas y situaciones. No existe en ella con propiedad ni simplicidad en un tiempo, ni identidad a lo largo de los momentos direrentes, sea cual sea la inclinación natural que nos lleve a imaginar esa simplicidad e identidad." 
le atribuía a la primera. La conciencia de la existencia de los objetos exteriores se muestra como lo más originario a partir de lo cual es posible tomar conciencia del fluir de nuestros estados internos y de nuestra existencia misma. No hay experiencia interna sin experiencia externa. No podemos ser conscientes de nosotros mismos si no somos, a la vez, conscientes de algo que no somos nosotros. Queda establecida pues, a partir del argumento de la refutación del idealismo, una estrecha relación de dependencia del sentido interno respecto del externo fundada particularmente en los caracteres temporales que cada uno de ellos presentan respectivamente. La sucesión de nuestros estados internos no puede, en efecto, determinarse como tal a menos que exista un marco de referencia permanente de objetos en el espacio. Así pues, si bien los objetos físicos son siempre objetos representados, lo son en un orden temporal que es irreductible al de nuestras representaciones internas por las cuales nos volvemos empíricamente autoconscientes de nuestra propia existencia.

\section{La noción de experiencia interna implícita en el argumento}

El argumento de la "Refutación..." supera, como acabamos de ver en el punto anterior, algunas dificultades que presentaba el planteo de esta misma cuestión en el capítulo de los "Paralogismos de la razón pura", particularmente el de la posibilidad de reducir el sentido externo al interno.

Creemos, sin embargo, que Kant no llevó hasta sus últimas consecuencias las interesantes consideraciones que allí realiza respecto de los caracteres propios de la experiencia interna y la relación que la misma guarda con el conocimiento de los objetos físicos.

El núcleo de la argumentación reside, a nuestro entender, en el significado de aquellas premisas en las que se describe el modo en que somos empíricamente autoconscientes de nosotros mismos. Kant comienza por establecer que somos conscientes de nuestra propia existencia como algo determinado en el tiempo, es decir, que aparecemos fenoménicamente ante nosotros mismos por medio de una multiplicidad sensible dada en el sentido interno bajo la forma del tiempo. También los objetos físicos se nos hacen presentes de un modo fenoménico. Sin embargo, dice Kant, en A 381-382, que los fenómenos externos proporcionan un sustrato como base de sus determinaciones transitorias. Por el contrario, el tiempo, como forma del sentido interno, sólo ofrece a nuestro conocimiento el cambio de las determinaciones, pero no el objeto determinable por ellas (cfr. loc. cit.). La temporalidad propia de nuestros estados internos tiene pues la peculiaridad de presentar este contenido intuitivo siempre en forma sucesiva. No hay permanencia en el sentido interno y es precisamente esta ausencia.de permanencia lo que constituye la clave de la argumentación. 
Ahora bien, cabría preguntarse en este punto si esta autoconciencia empírica de nuestra existencia, dada por medio del sentido interno, constituye o no un genuino autoconocimiento. En su libro Kant's Transcendental Idealism, $\mathrm{H}$. Allison propone, por ejemplo, una interesante interpretación de la refutación del idealismo según la cual la autoconciencia empírica no debe entenderse como un mero darse cuenta (awareness) de las representaciones propias, sino como un conocimiento empírico real del yo (mente) y sus estados. ${ }^{5}$ El contenido de esta experiencia interna son las representaciones tomadas como "objetos subjetivos". Las mismas son experimentadas como algo que existe objetivamente en el tiempo y como algo que tiene en él determinada posición temporal. El yo, por otra parte, es consciente de sí mismo como el poseedor de esta secuencia de representaciones y el sujeto de esta historia mental particular, lo cual le proporciona una identidad propia como sujeto empírico. Así pues, en la medida en que es consciente de la secuencia determinada de sus representaciones en el tiempo, es también consciente de su propia existencia como algo determinado en el tiempo.

Ahora bien, esta determinación temporal supone, según lo establece la primera analogía de la experiencia, algo permanente en la percepción. Como esto no puede encontrarse en la experiencia interna, lo permanente debe ser un objeto en el espacio. Allison subraya que para refutar el idealismo cartesiano no es suficiente establecer que debemos tener representaciones externas, sino que además es necesario demostrar que las mismas no son meramente imaginadas o soñadas. Para esto es indispensable, según su interpretación, partir de la tesis que mencionamos recién, es decir, que es preciso suponer que la autoconciencia de la propia existencia como algo determinado en el tiempo constituye una auténtica experiencia interna $\mathrm{o}$, dicho con otras palabras, que existe un genuino autoconocimiento. El cartesiano, conjetura Allison, no dudaría en aceptar esta tesis, pero una vez aceptada, el idealismo problemático se vuelve contra sí mismo. Si la autoconciencia empírica de nuestra existencia constituye conocimiento, entonces nuestros estados internos se encuentran en el tiempo objetivo y si esto es así, también debe encontrarse en el tiempo objetivo el objeto permanente que hace posible la determinación sucesiva de tales estados. Los objetos en el espacio no pueden ser, por tanto, meramente imaginados o soñados. El orden objetivo de representaciones internas supone, dadas sus características temporales, un orden igualmente objetivo de representaciones externas. Tanto unas como otras pertenecen a un mismo tiempo objetivo y constituyen pues dos polos de una única experiencia.

Creemos que esta interpretación de Allison es sumamente significativa desde el momento en que pone de relieve que el idealismo problemático

5 Cfr. H.E. Allison, Kant's Transcendental Idealism, Yale University Press, New Haven/Londres, 1983, pp. 294-309. 
queda estrictamente refutado sólo si se demuestra que nuestras representaciones externas son objetivas. Esto, que a nuestro entender es la clave del argumento, no se encuentra explicitado en el texto. Más bien Allison lo deduce a partir de su tesis de que la autoconciencia empírica de nuestra propia existencia constituye un conocimiento objetivo. Creemos, sin embargo, que esta última tesis es difícil de sostener en el marco de la prueba que Kant propone. En efecto, allí se establece que en el sentido interno no hay permanencia. El yo empírico no puede pues constituirse a la manera de un objeto sustancial cuyas determinaciones sean los diferentes estados de conciencia en los cuales se encuentra, porque si así fuera él mismo funcionaría como sustrato con respecto al cual estos estados internos se determinarían sucesivos. No sería, por tanto, necesario buscar tal sustrato en los objetos espaciales y la prueba, en consecuencia, se derrumbaría. El argumento más bien parece sugerir que las características temporales con que se presenta la multiplicidad interna impiden, o al menos dificultan, la constitución de un objeto del sentido interno que sea sustancial o, precisamente, un objeto al cual pueda aplicársele cualquiera de las categorías de relación, ya que la categoría de sustancia es condición de posibilidad de la aplicación de las categorías de causalidad y acción recíproca (cfr. A $187=$ B 230). Si esto es así, resulta difícil seguir considerando la autoconciencia empírica como una auténtica experiencia, ya que la multiplicidad interna mediante la cual nos autocaptamos sensiblemente no es subsumible bajo todas las categorías y no se ajusta, por tanto, a aquellas condiciones que hacen posible la objetividad misma. El sentido interno, lejos de dar lugar a un auténtico autoconocimiento, más bien parece mostrarse insuficiente para la constitución de un conocimiento objetivo, y sería esta dificultad la que en definitiva daría origen a su dependencia con respecto a la experiencia de los objetos en el espacio. Se rompe pues, en el contexto de la "Refutación...", el paralelismo entre la experiencia interna y la externa sobre el cual se apoyaba la argumentación de los paralogismos. Pero es precisamente esta ruptura y la relación asimétrica entre el sentido interno y el externo lo que permite, a nuestro entender, superar las dificultades que aquel argumento presentaba. En efecto, la diferencia esencial entre ambos en cuanto a la posibilidad de aplicar las categorias a sus respectivos contenidos intuitivos deja abierto el camino para establecer una distinción entre la experiencia externa y la interna que no pase simplemente por los caracteres sensibles con que se presentan los fenómenos (en un caso espacio-temporalmente, en el otro sólo temporalmente), sino que permita diferenciar entre un orden objetivo $y$ otro meramente subjetivo de representaciones. El idealismo cartesiano quedará refutado en la medida en que se logre evitar aquella postura subjetivista de la que hablamos en el primer punto, es decir, en cuanto se demuestre que aun siendo toda realidad siempre una realidad representada, es posible, de todos modos, distinguir entre la mera representación, como estado subjetivo de conciencia, y la 
representación de un mundo que es el mismo para todos. Y esta distinción, dentro del marco del idealismo trascendental y partiendo de la tesis de la incognoscibilidad de la cosa en sí, sólo podrá establecerse teniendo en cuenta alguna diferencia en el modo en que se aplican respectivamente las categorías a cada uno de estos ámbitos.

Debemos reconocer, sin embargo, que no está explícita en el texto la idea de que el contraste entre los caracteres temporales de la experiencia externa y los de la interna conduce a una asimetría en el modo de aplicación de las categorías y, por tanto, a la posibilidad de distinguir entre un orden objetivo y uno meramente subjetivo de representaciones. Creemos que Kant no llevó hasta sus últimas consecuencias la tesis de la ausencia de permanencia en el sentido interno. Al menos no lo hizo en el texto mismo de la "Refutación...". Pero llama notablemente la atención que en otro pasaje, agregado también en la segunda edición de la $\mathrm{KrV}^{6}{ }^{6}$ establece expresamente que para comprender la posibilidad de las cosas en conformidad con las categorías y demostrar así la realidad objetiva de las últimas, necesitamos intuiciones que sean en todos los casos externas (cfr. B 291). ${ }^{7}$ Las palabras de Kant sugieren que sólo en el ámbito de las representaciones espaciotemporales las categorías darían lugar a un conocimiento estrictamente objetivo. El pasaje concluye con la afirmación de que estas consideraciones son de gran importancia no sólo en tanto confirman lo establecido en la "Refutación...", sino también en cuanto marcan los límites de la posibilidad del conocimiento de nosotros mismos por medio del sentido interno (cfr. B 293-294).

De todos modos, aun en el mismo texto de la "Refutación..." aparece al menos sugerida la idea de que es necesario distinguir entre un orden subjetivo y uno objetivo para poder refutar el idealismo cartesiano. En efecto, Kant establece que "la percepción de lo permanente es posible sólo a través de una cosa fuera de mí, y no por la mera representación de una cosa fuera de mi" (B 275). Teniendo en cuenta los presupuestos del idealismo trascendental, no se puede, de ningún modo, entender este pasaje en un sentido realista. Creemos que la única interpretación posible es que Kant se está refiriendo efectivamente a la distinción entre un orden subjetivo y uno objetivo: en el orden de las meras representaciones, es decir, en el orden de las representaciones entendidas como estados subjetivos de conciencia no es posible encontrar nada permanente porque las mismas se hallan en continuo flujo. Lo permanente debe pertenecer a un mundo

6 Nos referimos a la "Nota general sobre el sistema de los principios" agregada al final del capítulo sobre los "Postulados del pensamiento empírico".

7 Teniendo en cuenta esta tesis que aparece en la "Nota general...", G.E. Franzwa propone una reformulación de la doctrina del esquematismo en la cual la esquematización de las categorías presupondría tanto el espacio como el tiempo (cfr. G.E. Franzwa, "Space and Schematism", Kant-Studien, no. 69, 1978, pp. 149-159). 
representado pero, a la vez, intersubjetivo e irreductible a la sucesión de mis estados internos, ya que son estos últimos los que dependen de él para constituirse en forma de serie.

La "Refutación del idealismo" establece pues, según la interpretación que hemos propuesto, no sólo la irreductibilidad del orden temporal propio de los objetos espaciales al orden temporal de nuestros estados internos, sino más aún la irreductibilidad del ámbito de las representaciones del mundo físico -es decir, de un mundo estrictamente objetivo e intersubjetivo- al ámbito de nuestras representaciones entendidas como meros estados subjetivos de conciencia.

Si esto es así, el sentido interno daría lugar a una autoconciencia empírica de nosotros mismos a partir del material sensible que él ofrece; pero no daría lugar, en rigor, a un auténtico autoconocimiento. El fluir de nuestros estados subjetivos se mostraría siempre como una contracara interna de la experiencia propiamente dicha, es decir, como una serie que, incapaz de constituirse en forma autónoma, de algún modo se "sostendría" en la permanencia de un mundo físico representado pero no por ello reducible al contenido de nuestra vida psíquica. 